\title{
Kaempferol induces ATM/p53-mediated death receptor and mitochondrial apoptosis in human umbilical vein endothelial cells
}

\author{
CHIU-FANG LEE ${ }^{1 *}$, JAI-SING YANG $^{2 *}$, FUU-JEN TSAI $^{3,4}$, NI-NA CHIANG $^{1}$, \\ CHI-CHENG LU ${ }^{5}$, YU-SYUAN HUANG ${ }^{6}, \mathrm{CHUN} \mathrm{CHEN}^{6}$ and FU-AN CHEN ${ }^{6}$
}

\author{
${ }^{1}$ Kaohsiung Veterans General Hospital Pingtung Branch, Pingtung; ${ }^{2}$ Department of Medical Research, \\ China Medical University Hospital, China Medical University, Taichung; ${ }^{3}$ Human Genetic Center, \\ China Medical University Hospital, Taichung; ${ }^{4}$ School of Post-Baccalaureate Chinese Medicine, \\ China Medical University, Taichung; ${ }^{5}$ School of Nutrition and Health Sciences, Taipei Medical University, \\ Taipei; ${ }^{6}$ Department of Pharmacy and Master Program, Tajen University, Pingtung, Taiwan, R.O.C.
}

Received December 22, 2015; Accepted January 30, 2016

DOI: $10.3892 /$ ijo.2016.3420

\begin{abstract}
Kaempferol is a member of the flavonoid compounds found in vegetables and fruits. It is shown to exhibit biological impact and anticancer activity, but no report exists on the angiogenic effect of kaempferol and induction of cell apoptosis in vitro. In this study, we investigated the role of kaempferol on anti-angiogenic property and the apoptotic mechanism of human umbilical vein endothelial cells (HUVECs). Our results demonstrated that kaempferol decreased HUVEC viability in a time- and concentration-dependent manner. Kaempferol also induced morphological changes and sub-G1 phase cell population (apoptotic cells). Kaempferol triggered apoptosis of HUVECs as detecting by DNA fragmentation, comet assay and immunofluorescent staining for activated caspase-3. The caspase signals, including caspase- $8,-9$ and -3 , were time-dependently activated in HUVECs after kaempferol exposure. Furthermore, pre-treatment with a specific inhibitor of caspase-8 (Z-IETD-FMK) significantly reduced the activity of caspase- $8,-9$ and -3 , indicating that extrinsic pathway is a major signaling pathway in kaempferoltreated HUVECs. Importantly, kaempferol promoted reactive oxygen species (ROS) evaluated using flow cytometric assay in HUVECs. We further investigated the upstream extrinsic pathway and showed that kaempferol stimulated death receptor signals [Fas/CD95, death receptor 4 (DR4) and DR5] through increasing the levels of phosphorylated p53
\end{abstract}

Correspondence to: Professor Fu-An Chen, Department of Pharmacy and Master Program, Tajen University, 20 Weixin Road, Yanpu, Pingtung 90741, Taiwan, R.O.C.

E-mail: fachen.tajen@yahoo.com.tw

*Contributed equally

Key words: kaempferol, human umbilical vein endothelial cells, apoptosis, extrinsic pathway, reactive oxygen species, p53/ATM/ death receptor signaling and phosphorylated ATM pathways in HUVECs, which can be individually confirmed by $\mathrm{N}$-acetylcysteine (NAC), ATM specific inhibitor (caffeine) and p53 siRNA. Based on these results, kaempferol-induced HUVEC apoptosis was involved in an ROS-mediated p53/ATM/death receptor signaling. Kaempferol might possess therapeutic effects on cancer treatment in anti-vascular targeting.

\section{Introduction}

Angiogenesis is an important physiological process during promoting tumor growth or in metastatic tumors (1). Suppression of endothelial cell proliferation or induction of cell apoptosis is a good strategy for blocking tumor angiogenesis (2). The human umbilical vein endothelial cells (HUVECs) are the most widely used endothelial cell model, which can be examined through many processes for anti-angiogenic actions (3). Moreover, the induction of endothelial cell apoptosis is one of the central antiangiogenic mechanisms $(3,4)$. Two major important pathways contribute to the apoptotic processes, including the intrinsic mitochondria-mediated pathway and the extrinsic death receptor signaling (5). Mitochondrial permeability can be regulated to release various apoptotic factors such as cytochrome $c$, Apaf- 1 and pro-caspase- 9 to cytosol to form apoptosome and to activate the downstream of caspase-9 $(6,7)$. The membrane death receptors (extrinsic apoptotic pathway) located in the membrane include Fas/ CD95, death receptor 4 (DR4) and DR5 that can influence the distal executioner caspases (6). In addition, reactive oxygen species (ROS) production and DNA damage caused by anticancer drugs lead to an increase of phosphorylation of ataxia-telangiectasia-mutated kinase (ATM) and p53 to trigger human cancer cell apoptosis (8). p53 phosphorylation on the residue of Ser15 has been linked to apoptosis and shown to be a transcription factor to modulate apoptotic target genes such as Fas and DR5 (9). p53 gene expression has been shown to upregulate both the extrinsic and the intrinsic apoptotic signaling pathways $(6,10)$.

Kaempferol is a dietary flavonoid and is found in fruits and vegetables and in traditional Chinese medicines $(11,12)$. 
The pharmacological activities of kaempferol were reported to exhibit anti-inflammatory, antioxidant, cardio-protective and antitumor activities (12). Our previous study demonstrated that kaempferol-induced apoptosis in human osteosarcoma cells is mediated through endoplasmic reticulum stress and mitochondria-dependent signaling (13). Kaempferol also induces autophagy by AMPK and AKT signaling and causes $\mathrm{G} 2 / \mathrm{M}$ phase arrest via downregulation of CDK1/cyclin B in human hepatocarcinoma cells (14). However, there is no report addressing the possible anti-angiogenetic mechanism of kaempferol. The objective of the current study was to explore apoptotic evidence and its underlying molecular mechanism induced by kaempferol in HUVECs. Kaempferol might induce both the extrinsic and the intrinsic apoptotic pathways in HUVEC cells through ROS-mediated p53/ATM/death receptor signaling.

\section{Materials and methods}

Chemicals and reagents. Caffeine, 4,6-diamidino2-phenylindole dihydrochloride (DAPI), kaempferol, 3-(4,5-dimethylthiazol-2-yl)-2,5-diphenyltetrazolium bromide (MTT), N-acetylcysteine (NAC) and propidium iodide (PI) were purchased from Sigma-Aldrich (St. Louis, MO, USA). Medium 200, Low Serum Growth Supplement (LSGS), TrypsinEDTA, 2,7-dichlorodihydrofluorescein diacetate ( $\mathrm{H}_{2}$ DCFDA) and Fluo-4/AM were purchased from Thermo Fisher Scientific (Carlsbad, CA, USA). Caspase-3, Caspase-8 and Caspase-9 Colorimetric Assay kits and caspase- 8 inhibitor Z-IETD-FMK were bought from R\&D Systems Inc. (Minneapolis, MN, USA). Primary antibodies [Fas/CD95, DR4, DR5, p-ATM (Ser1981), ATM and $\beta$-actin], horseradish peroxidase (HRP)-conjugated secondary antibodies against rabbit or mouse immunoglobulin and p53 siRNA were obtained from Santa Cruz Biotechnology (Santa Cruz, CA, USA). Anti-caspase-3, -8 and -9 antibodies were obtained from Cell Signaling Technology (Danvers, MA, USA). Antibodies against p53 and p-p53 (Ser15) were obtained from Abcam (Cambridge, Cambridgeshire, UK).

Cell culture. Human umbilical vein endothelial cells (HUVECs) were purchased from the Bioresource Collection and Research Center (BCRC, Hsinchu, Taiwan) and cultured in Medium 200 plus LSGS at $37^{\circ} \mathrm{C}$ in a humidified atmosphere with $5 \% \mathrm{CO}_{2}$. The cells were used between the second to fifth passages.

Cell viability. HUVECs were plated onto 96-well microplates at a density of $5 \times 10^{3}$ cells $/ 100 \mu 1$ per well and then incubated with kaempferol at the concentrations of $0,50,100,150$ and $200 \mu \mathrm{M}$ for 24,48 and 72 -h treatment. Cell viability was determined by MTT assay as previously described (15), and the optical density ratio of the treatment to the control (\% of control) was calculated.

Cell morphological detection and DNA content analysis by flow cytometry. HUVECs were treated with $100 \mu \mathrm{M}$ kaempferol for 24 and $48 \mathrm{~h}$. The cells were examined and photographed using a phase-contrast microscope. Then the cells were collected andfixed in $75 \%$ ethanol overnight at $-20^{\circ} \mathrm{C}$ before being stained with $0.1 \mathrm{M}$ phosphate/citric acid buffer (0.2 $\mathrm{M} \mathrm{NaHPO}_{4}$ and 0.1 $\mathrm{M}$ citric acid, $\mathrm{pH} 7.8$ ) and $40 \mu \mathrm{g} / \mathrm{ml}$
PI for $30 \mathrm{~min}$ at room temperature in the dark. The cells were determined with BD FACSCalibur (BD Biosciences, Franklin Lakes, NJ, USA) as previously described (6).

DAPI staining and comet assay. HUVECs were treated with $100 \mu \mathrm{M}$ kaempferol for $48 \mathrm{~h}$. The cells were fixed and incubated with $1 \mu \mathrm{g} / \mathrm{ml}$ DAPI following a previously reported method $(6,16)$. After being harvested, cells were combined with molten low-melting agarose (Sigma-Aldrich) at a density of $1 \times 10^{5}$ cells $/ \mathrm{ml}$. The agarose-cell mixture $(50 \mu \mathrm{l})$ was immediately pipetted onto comet slides. The slides were then immersed in pre-chilled lysis solution for $30 \mathrm{~min}$ at $4^{\circ} \mathrm{C}$ as previously described (10). After lysis, horizontal electrophoresis was performed for $30 \mathrm{~min}$ at $300 \mathrm{~mA}$. The slides were fixed by $70 \%$ ethanol for 5 min before being stained with $50 \mu \mathrm{l}$ nuclear counterstain DAPI solution (final concentration: $1 \mu \mathrm{g} / \mathrm{ml}$ ) and viewed under a fluorescence microscope.

Immunofluorescence staining. HUVECs $\left(5 \times 10^{4}\right.$ cells/well) on 4-well chamber slides were treated with $100 \mu \mathrm{M}$ kaempferol for $24 \mathrm{~h}$. Cells were fixed in 3\% formaldehyde (Sigma-Aldrich) for $15 \mathrm{~min}$, permeabilized with $0.1 \%$ Triton-X 100 in PBS for $1 \mathrm{~h}$ with blocking of non-specific binding sites using $2 \%$ bovine serum albumin (BSA) as previously described $(6,17)$. These fixed cells were stained with cleaved caspase- 3 antibody (1:100 dilution, Cell Signaling Technology) overnight before being detected using a goat anti-mouse IgG secondary antibody conjugated fluorescein isothiocyanate (FITC) (1:500 dilution, green fluorescence) (Merck Millipore, Billerica, MA, USA), followed by nuclei counterstaining using and PI (red fluorescence). Images were collected with a Leica TCS SP2 Confocal Spectral Microscope (Leica Microsystems, Heidelberg, Mannheim, Germany)

Determination of caspase-3/-8/-9 activities and effects of their specific inhibitors. HUVECs $\left(5 \times 10^{6}\right.$ cells) were pretreated with or without $10 \mu \mathrm{M}$ Z-IETD-FMK (a specific caspase- 8 inhibitor) for $1 \mathrm{~h}$ and incubated in 75-T flasks and treated with kaempferol for 24 and $48 \mathrm{~h}$. After treatment, cells were harvested and lysed, and cell lysates (50 $\mu \mathrm{g}$ proteins) were incubated to check relative caspase activity using Caspase-3, Caspase- 8 and Caspase-9 Colorimetric Assay Kits (R\&D Systems Inc.) following the manufacturer's instructions.

Measurements of intracellular $\mathrm{Ca}^{2+}$ levels and mitochondrial membrane potential $(\Delta \Psi m)$. HUVECs were treated with $100 \mu \mathrm{M}$ kaempferol for 6, 12 and $24 \mathrm{~h}$. Cells were then harvested and labeled with $2 \mu \mathrm{M}$ Fluo-4/AM (a specific intracellular $\mathrm{Ca}^{2+}$ fluorescence probe) and $500 \mathrm{nM} \mathrm{DiOC}_{6}(3)$, respectively, at $37^{\circ} \mathrm{C}$ for $30 \mathrm{~min}$. Consequently, intracellular $\mathrm{Ca}^{2+}$ and $\Delta \Psi \mathrm{m}$ were individually analyzed for fluorescence intensity by flow cytometry as previously described (17).

Western blot analysis. HUVECs $\left(5 \times 10^{6}\right.$ cells) were incubated in $100 \mu \mathrm{M}$ kaempferol for 0,12 or $24 \mathrm{~h}$. After being harvested and lysed, the $10 \%$ SDS-polyacrylamide electrophoresis (SDS-PAGE) gels were used to separate equal amount of protein extract from cell lysate as detailed by Yang et al (18). The appropriate the primary antibodies were hybridized to observe the specific protein signals. Then the HRP-conjugated 


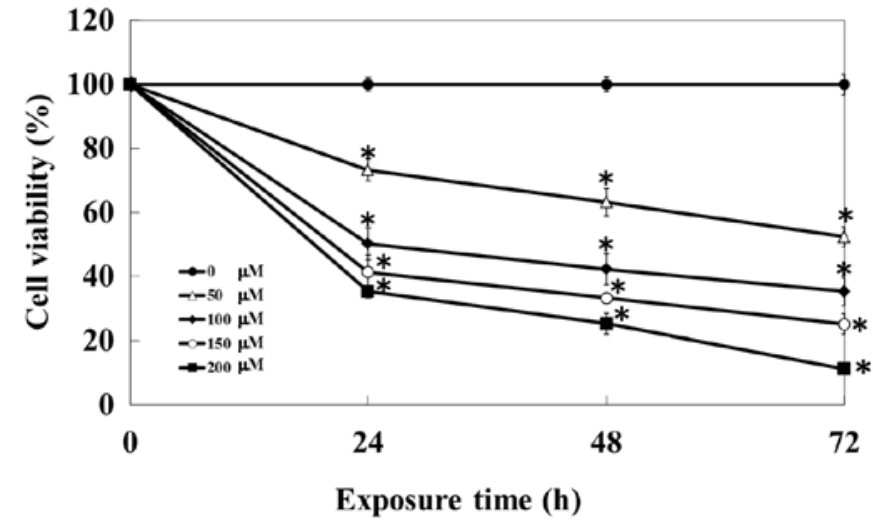

Figure 1. Kaempferol reduces cell viability in HUVECs. Cells were exposed to various concentrations of kaempferol $(0,50,100,150$ or $200 \mu \mathrm{M})$ for 24, 48 and $72 \mathrm{~h}$ and determined and analyzed the viability using the MTT assay. The values are presented as the means $\pm S D(n=3)$. ${ }^{*} \mathrm{P}<0.05$ versus untreated control.

secondary antibodies were applied before using Immobilon Western HRP substrate kit (Merck Millipore). The densitometric quantification of each band was performed utilizing NIH ImageJ 1.47 software.

Measurements of ROS production after $N$-acetylcysteine and caffeine pre-treatment for cell viability. HUVECs were treated with $100 \mu \mathrm{M}$ kaempferol for 6,12 and $48 \mathrm{~h}$. Cells were then harvested and labeled with $20 \mu \mathrm{M} \mathrm{H}_{2}$ DCFDA (a specific ROS fluorescent probe) at $37^{\circ} \mathrm{C}$ for $30 \mathrm{~min}$. Consequently, ROS was analyzed for fluorescence intensity by flow cytometry as previously described (19). Cells were incubated with $100 \mu \mathrm{M}$ kaempferol for $48 \mathrm{~h}$ before individual pretreatment with or without the $10 \mathrm{mM} \mathrm{N}$-acetylcysteine (NAC, an antioxidant) or $1 \mathrm{mM}$ caffeine (an ATM kinase inhibitor) for $1 \mathrm{~h}$. After that, cells were determined for cell viability by MTT assay as described above.

Small interference RNA transfection. HUVEC cells were grown to $70 \%$ confluence in 6-well culture plates, and control siRNA $(100 \mathrm{nM})$ or p53 siRNA $(100 \mathrm{nM})$ was transfected using Lipofectamine 2000 (Thermo Fisher Scientific) according to the manufacturer's instructions. After transfection, cells were seeded and thereafter exposed to $100 \mu \mathrm{M}$ kaempferol for $48 \mathrm{~h}$ before analyses using western blot and MTT assay, respectively.

Statistical analysis. The data represent the mean \pm standard deviation (SD) from at least three separate experiments. Statistical analysis was carried out using Student's t-test, and $\mathrm{P}<0.05$ was considered statistically significant.

\section{Results}

Kaempferol induces growth inhibition in HUVECs. At first, our study focused on the growth inhibition effects of kaempferol on HUVECs. Cells were treated with 0, 50, 100, 150 and $200 \mu \mathrm{M}$ of kaempferol, and cell number was counted at 24,48 and $72 \mathrm{~h}$. Our results showed that kaempferol decreased viable HUVECs in a concentration- and time-dependent manner

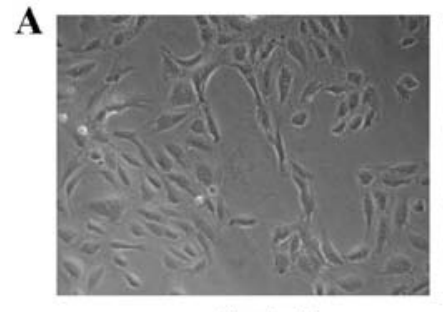

Control

B

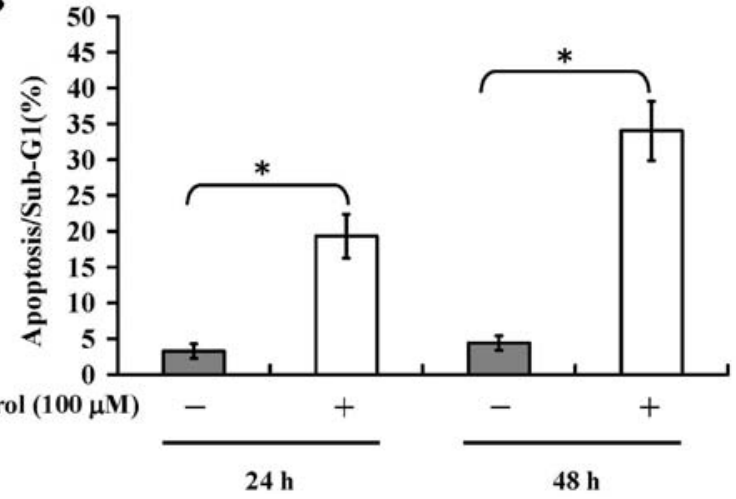

Figure 2. Kaempferol induces morphological changes and hypodiploid DNA contents in HUVECs. (A) Cells in response to $100 \mu \mathrm{M}$ kaempferol for a $24-\mathrm{h}$ exposure were photographed at $\times 20$ magnification and showed apoptotic morphological changes. (B) Cells were treated with $100 \mu \mathrm{M}$ of kaempferol for 24 and $48 \mathrm{~h}$. Cells with hypodiploid DNA contents (\%) represent the fractions undergoing apoptotic DNA degradation by flow cytometry and the Modfit program. The values are presented as the means \pm SD $(n=3)$. ${ }^{*} P<0.05$ versus untreated control.

(Fig. 1). The $\mathrm{IC}_{50}$ of kaempferol was $103.25 \pm 4.15 \mu \mathrm{M}$ after 24-h treatment.

Kaempferol triggers morphological changes and apoptosis in HUVECs. To understand whether apoptotic mechanisms are involved in kaempferol-treated HUVECs, the morphological changes and DNA content using flow cytometric analysis were investigated. Our results showed that HUVECs were detached from the surface of the plate and showed shrinkage in kaempferol-treated cells (Fig. 2A, right), and the control group showed normal morphology (Fig. 2A, left). The results from the DNA content demonstrated that kaempferol induced an increase of hypodiploid apoptotic cell population (sub-G1 phase) at 24 and $48 \mathrm{~h}$ treatments (Fig. 2B). These effects are time-dependent. Our results indicated that kaempferol provoked apoptotic cell death in HUVECs.

Kaempferol induces DNA condensation, DNA damage and caspase-3 protein expression in HUVECs. To confirm the apoptotic evidence in kaempferol-treated HUVECs, DAPI stain for DNA condensation and comet assay for DNA damage were monitored. Our results showed that kaempferol induced DNA condensation (Fig. 3A) and DNA damage (Fig. 3B) in HUVECs cells. It is well known that caspase-3 is a key mediator of cell apoptosis $(13,14)$. Next, we used caspase-3 immunofluorescence staining and confocal laser scanning microscopy to observe the caspase- 3 protein expression. The caspase-3 protein expression (green color) was observed in the cytosol of kaempferol-treated HUVECs (Fig. 3C). Our results 
$\mathbf{A}$

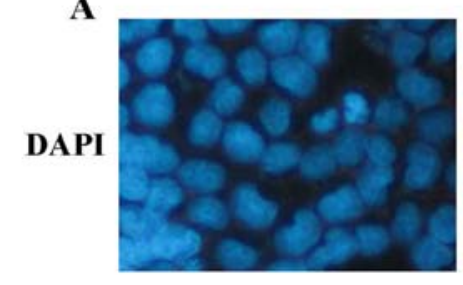

Control

B
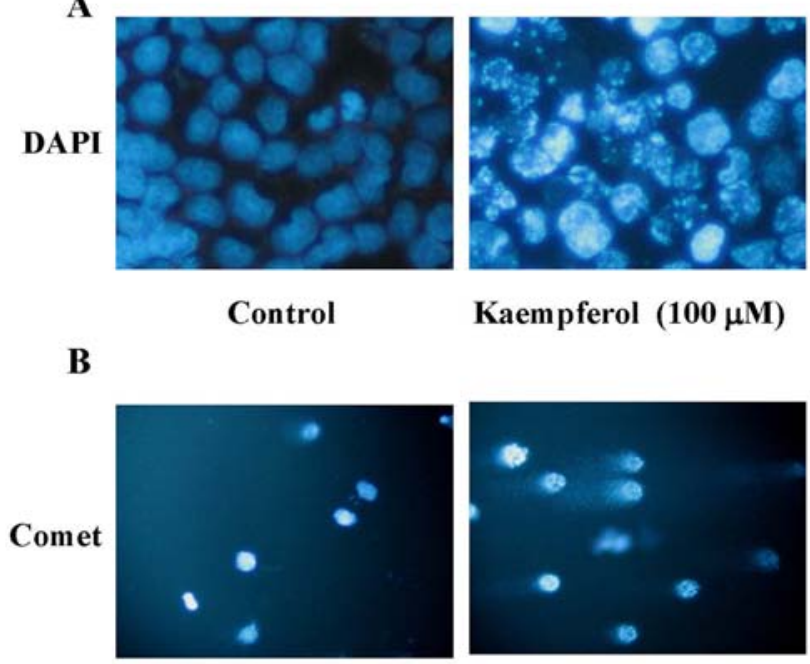

Control

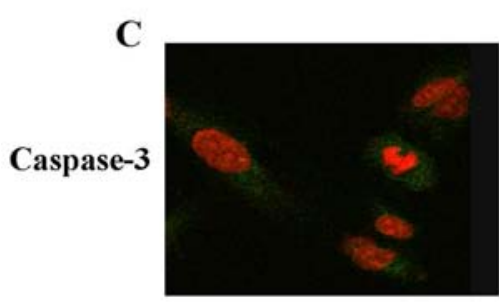

Control

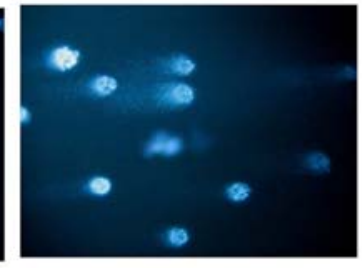

Kaempferol $(100 \mu \mathrm{M})$

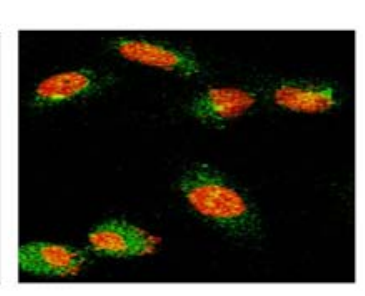

Kaempferol $(100 \mu \mathrm{M})$

Figure 3. Kaempferol induces DNA condensation, DNA damage and caspase-3 protein expression in HUVECs. Cells were treated with $100 \mu \mathrm{M}$ kaempferol for $48 \mathrm{~h}$. (A) DAPI staining to analyze chromatin condensation (a catachrestic of apoptosis). (B) Comet assay to analyze DNA damage. The images were observed and captured using a fluorescent microscope. (C) Kaempferol stimulated the translocation of caspase-3 trafficking to nuclei in HUVECs by confocal laser scanning microscope as described in Materials and methods.

demonstrated that kaempferol provoked apoptotic cell death through DNA damage and caspase-3 activation in HUVECs.

Kaempferol stimulates intracellular $\mathrm{Ca}^{2+}$ levels and loss of $\triangle \Psi m$ in HUVECs. To determine the roles of intracellular $\mathrm{Ca}^{2+}$ levels and $\Delta \Psi \mathrm{m}$ levels of apoptotic death induced by kaempferol, we detected the intracellular $\mathrm{Ca}^{2+}$ by Fluo-4/AM dye and $\Delta \Psi \mathrm{m}$ by $\mathrm{DiOC}_{6}(3)$ dye at 6,12 and $24 \mathrm{~h}$, respectively. Kaempferol increased intracellular $\mathrm{Ca}^{2+}$ levels (Fig. 4A) and depletion of $\Delta \Psi \mathrm{m}$ (Fig. 4B) in HUVECs. The data indicated that kaempferol-provoked apoptotic death in HUVECs might be mediated through $\mathrm{Ca}^{2+}$ signal and mitochondrial pathway.

Kaempferol induces the activity of caspase- $8,-9$ and -3 in HUVECs. To determine the major caspase pathway of apoptotic death induced by kaempferol, we further detected the activity of caspase- $8,-9$ and -3 at 24 and $48 \mathrm{~h}$, respectively. Our data inducted that the activities of caspase- $8,-9$ and -3 were significantly increased in kaempferol-treated HUVECs in a time-dependent manner (Fig. 5A). These results suggested that both the intrinsic mitochondria-mediated pathway and the extrinsic death receptor signaling are involved in kaempferolinduced apoptosis in HUVECs. To confirm our suggestion, we used western blotting to detect the cleaved form of caspase-8,

$\mathbf{A}$

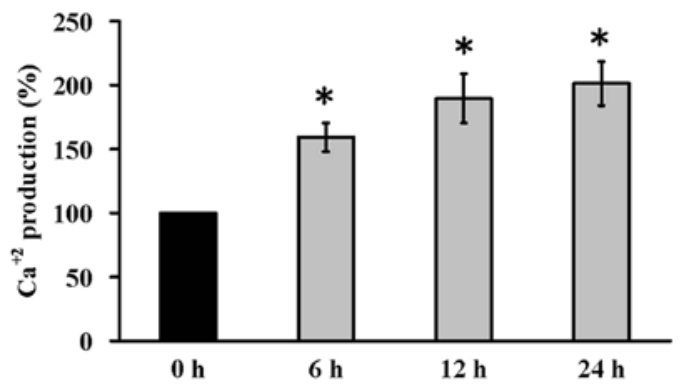

B

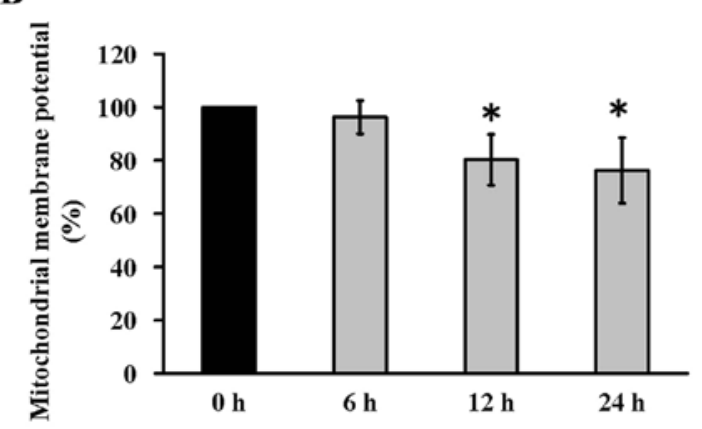

Figure 4. Kaempferol stimulates intracellular $\mathrm{Ca}^{2+}$ levels and loss of $\Delta \Psi \mathrm{m}$ in HUVECs. (A) Intracellular $\mathrm{Ca}^{2+}$ levels of HUVECs treated with $100 \mu \mathrm{M}$ kaempferol as determined by Fluo-4/AM fluorescent dye using flow cytometry analysis. (B) The levels of HUVECs after kaempferol treatment as determined by $\mathrm{DiOC}_{6}(3)$ fluorescent dye using flow cytometry analysis. The values are presented as the means $\pm \mathrm{SD}(\mathrm{n}=3)$. ${ }^{*} \mathrm{P}<0.05$ versus untreated control.

$\mathbf{A}$

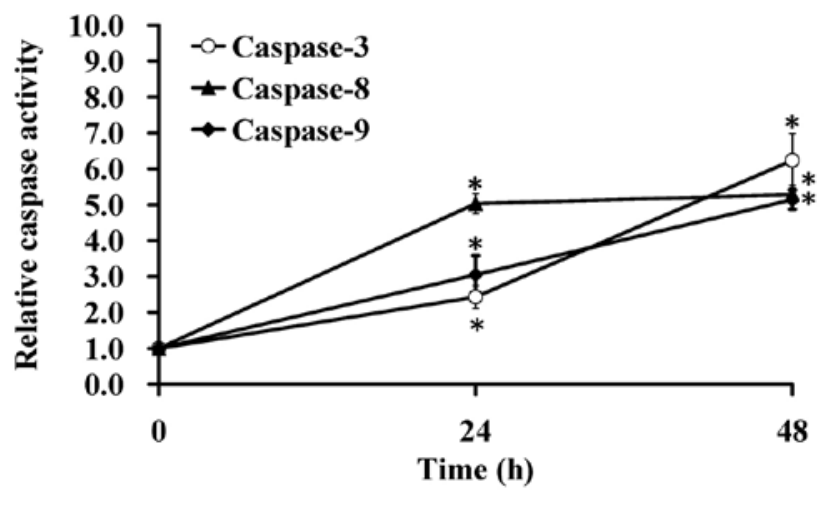

B

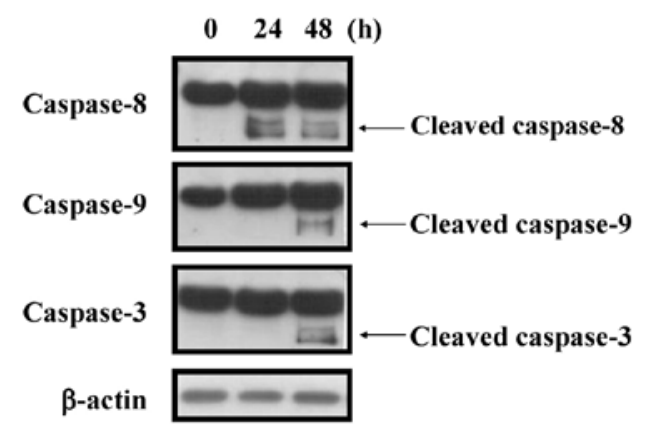

Figure 5. Kaempferol triggers caspase-8/-9/-3 activities and protein levels in HUVECs. Cells were treated with $100 \mu \mathrm{M}$ kaempferol for 0,24 and $48 \mathrm{~h}$. (A) Kaempferol-stimulated the activities of caspase-8, -9 and -3 in HUVECs were detected by colorimetric assay kits. The values are presented as the means $\pm \mathrm{SD}(\mathrm{n}=3) .{ }^{*} \mathrm{P}<0.05$ versus untreated control. (B) The total proteins were harvested and determined the protein levels of caspase- $8,-9$ and -3 by western blotting. $\beta$-actin served as a loading control. 

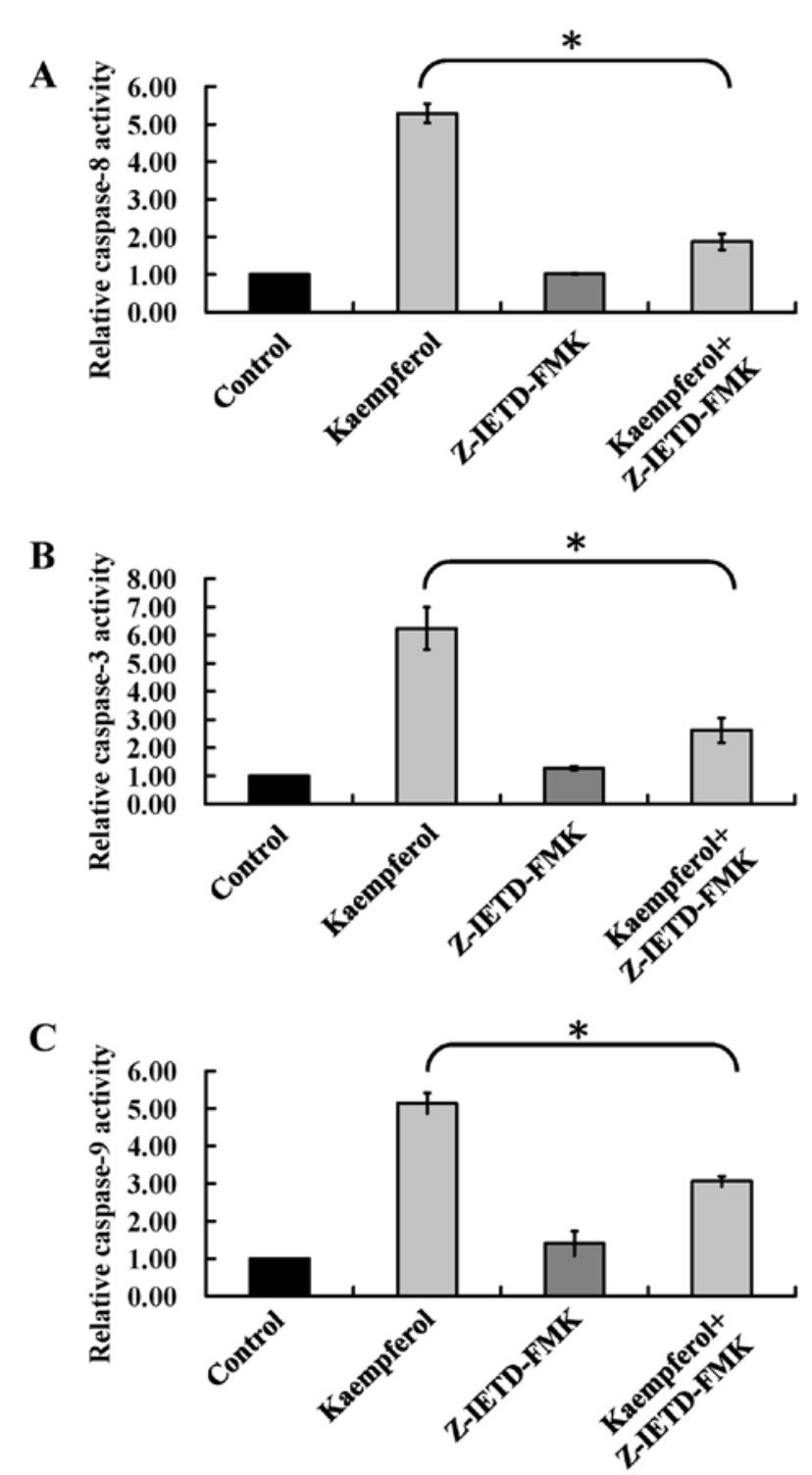

Figure 6. Caspase-8 inhibitor (Z-IETD-FMK) decreases kaempferol-induced caspase-8/-9/-3 activities in HUVECs. Cells were pretreated with $10 \mu \mathrm{M}$ caspase-8 inhibitor (Z-IETD-FMK) for $2 \mathrm{~h}$ prior to treatment with $100 \mu \mathrm{M}$ kaempferol for $48 \mathrm{~h}$. Cells were harvested for measuring the caspase-8 (A), caspase-9 (B) and caspase-3 (C) activities as described in Materials and methods. The values are presented as the means $\pm \mathrm{SD}(\mathrm{n}=3)$. ${ }^{*} \mathrm{P}<0.05$ versus kaempferol alone sample.

-9 and -3 . Our results showed that the cleaved caspase- $8,-9$ and -3 protein level were significantly increased in HUVECs prior to kaempferol challenge at $48 \mathrm{~h}$ (Fig. 5B). Strikingly, caspase- 8 activity was significantly increased at 24 -h treatment in treated HUVECs. The data indicated that extrinsic death receptor pathway is a key signal in kaempferol-induced apoptosis of HUVECs.

Z-IETD-FMK blocks the activity of caspase- $8,-9$ and -3 in kaempferol-treated HUVECs. Our hypothesis that kaempferolprovoked apoptosis is mediated mainly through extrinsic death receptor pathway. To prove our hypothesis, Z-IETD-FMK (a specific caspase- 8 inhibitor) was used to block caspase- $8,-3$, and -9 activities. Our results demonstrated that pre-incubation with the specific inhibitor of Z-IETD-FMK strongly decreased
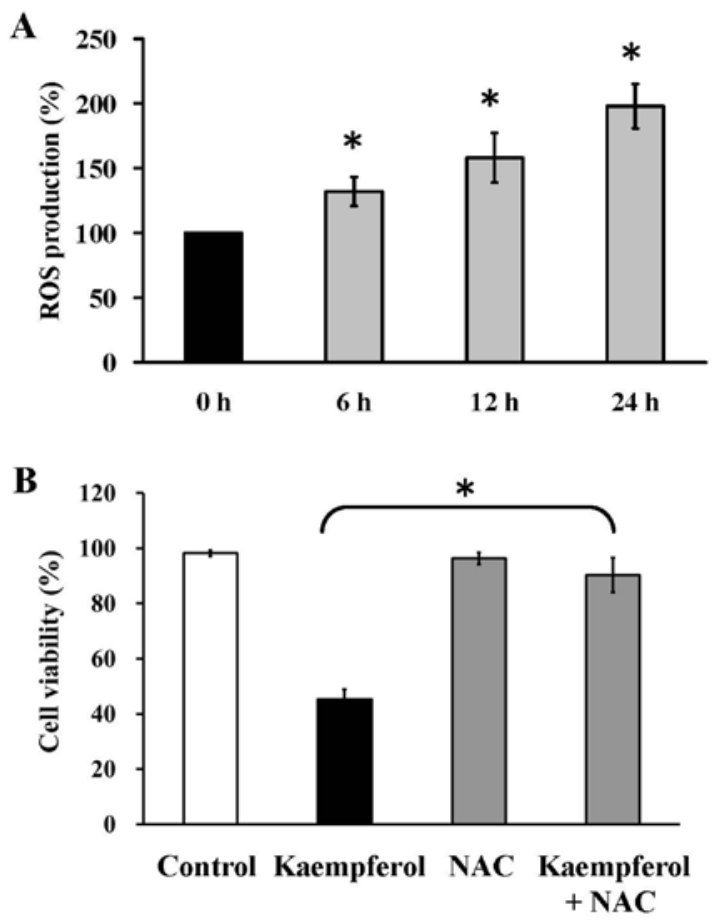

Figure 7. Kaempferol induces ROS generation and N-acetylcysteine reduces kaempferol-induced growth inhibition effect on HUVECs. (A) Treatment with $100 \mu \mathrm{M}$ kaempferol for $0,6,12$ and $24 \mathrm{~h}$ was subjected to ROS productions by flow cytometry. (B) Pre-treatment with NAC $(10 \mathrm{mM}$, a ROS scavenger) in kaempferol-treated HUVECs restored the cell viability by MTT assay. The values are presented as the means \pm SD $(n=3)$. ${ }^{*} \mathrm{P}<0.05$ versus untreated control or kaempferol alone sample.

the activity of caspase-8 (Fig. 6A), caspase-3 (Fig. 6B), and caspase-9 (Fig. 6C) compared with kaempferol treatment alone. Overall, these data demonstrated that extrinsic death receptor pathway is a crucial element in kaempferol-triggered apoptosis of HUVECs.

Kaempferol increases ROS generation and N-acetylcysteine reduces kaempferol-induced growth inhibition effect in HUVECs. Our findings showed that kaempferol increased intracellular ROS production at 6, 12 and $24 \mathrm{~h}$ in HUVECs by using flow cytometry and $\mathrm{H}_{2}$ DCFDA (a specific fluorescent probe) (Fig. 7A). Cells showed a significant inhibitory effect on kaempferol-induced growth inhibition after pre-treatment with NAC (Fig. 7B). These data indicated that ROS production is important in kaempferol-triggered apoptosis of HUVECs.

ATM-p53-mediated death receptor pathway is involved in kaempferol-induced apoptosis. It was reported that ROS can modulate death receptor pathway in cancer cells $(8,10)$. Our hypothesis showed that extrinsic death receptor pathway is a central component in kaempferol-triggered apoptosis of HUVECs. The results revealed that kaempferol stimulated the death receptor-associated protein levels, including Fas/CD95, DR4 and DR5 in HUVECs (Fig. 8A). It is well documented that p53 gene and its phosphorylation at the Ser15 interact Fas/ CD95 activation during cell apoptosis $(6,20)$. To elucidate the possible signaling pathway in kaempferol-provoked apoptosis, the levels of associated proteins were evaluated. Kaempferol increased the protein level of ATM, p53, phosphorylation of 
$\mathbf{A}$

B

$0 \quad 24 \quad 48(h)$

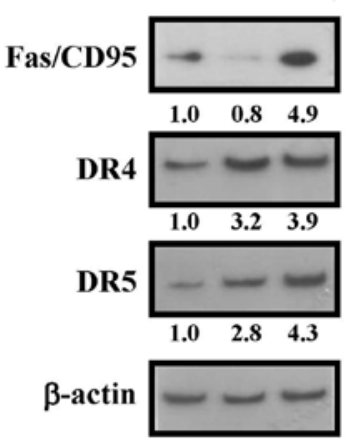

0 $\quad 24 \quad 48$ (h)

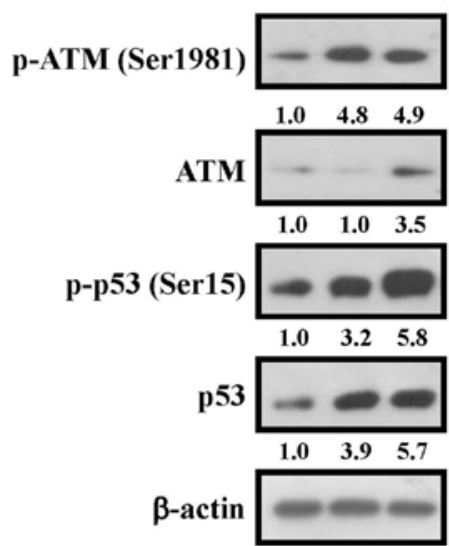

Figure 8. Kaempferol contributes to p53-correlated ATM/Fas/DR4/DR5 apoptotic signaling in HUVECs. Cells were treated with $100 \mu \mathrm{M}$ kaempferol for 0,24 and $48 \mathrm{~h}$, and total proteins were prepared and subjected to western blot analysis. The membranes were incubated with (A) anti-Fas/CD95, antiDR4 and anti-DR5 antibodies; (B) p-ATM (Ser1981), ATM, p-p53 (Ser15) and $\mathrm{p} 53$ antibodies. The blot was probed with anti- $\beta$-actin antibody to confirm equal loading. Each band was quantified using ImageJ software.

ATM and p53, followed by increasing levels of Fas, DR4 and DR5 based on the exposure time (Fig. 8B). Our results indicated that kaempferol increased the protein level of Fas/CD95, DR4 and DR5 through the ATM-p53-dependent regulation of transcription levels. We also re-checked the kaempferolcaused ATM-p53-dependent signal in HUVECs, and caffeine (an ATM kinase inhibitor) and p53 siRNA were used to block ATM and p53 function. Pre-treatment with caffeine reversed the inhibition of cell viability in treated group (Fig. 9A). Moreover, p53 siRNA also had a similar effect in kaempferoltreatment group (Fig. 9B). The results from our experimental approaches indicate that kaempferol-induced apoptosis of HUVECs is mediated through ATM-p53-mediated pathway.

\section{Discussion}

Kaempferol is a flavonol present in fruits and vegetables, including onions, kale, broccoli, apples, cherries, berries, tea and red wine $(11,12)$. Kaempferol has many biological properties, including anti-cancer, antioxidant activity, antiinflammatory effects $(12,21)$. Kaempferol induces apoptosis and cell cycle arrest in various cancer cell lines, including colon cancer (22), liver cancer (23), gastric cancer (24), and bladder cancer (25) cells. Kim et al (26) demonstrated that kaempferol can modulate angiogenesis and immune-endothelial cell adhesion. Zhao et al (27) showed that kaempferol from $\mathrm{Pu}$-erh tea has anti-cancer and anti-angiogenesis effects. Currently, the mechanism involved in the kaempferol anti-angiogenesis effects is unknown. In this study, we are the first to report that kaempferol induced growth inhibition (Fig. 1) and apoptosis (Figs. 2 and 3) in HUVEC cells. Our results also showed that kaempferol induced the activity of caspase-8, -9 and -3 (Fig. 4) and Fas/CD95, DR4 and DR5 at protein levels (Fig. 8) in HUVEC cells. Moreover, major cell signaling involved in kaempferol-treated HUVECs were investigated, we focused on the ROS-ATM-p53 signaling. Our results demonstrated that kaempferol induced ROS production (Fig. 7), ATM,
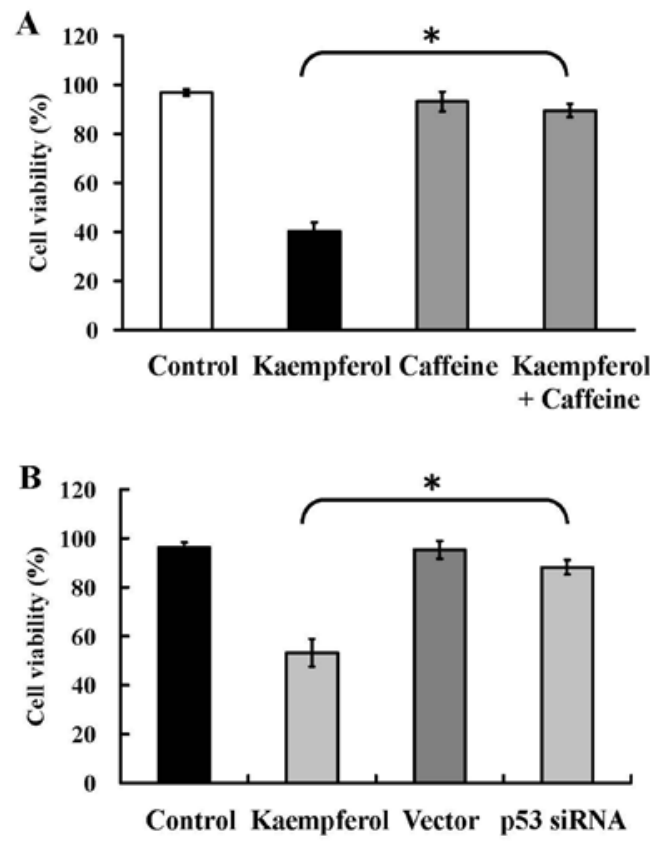

Figure 9. Caffeine and p53 siRNA reduce kaempferol-induced growth inhibition effect in HUVECs. (A) Cells were pretreated with or without $2 \mathrm{mM}$ caffeine (an ATM/ATR inhibitor) for $2 \mathrm{~h}$ and further incubated with $100 \mu \mathrm{M}$ kaempferol. After a 48-h exposure, cell viability was calculated by MTT assay. (B) HUVECs after being transfected with or without control vector or p53 siRNA were exposed to $100 \mu \mathrm{M}$ kaempferol. Transfected HUVECs following exposure to kaempferol for $48 \mathrm{~h}$ was determined for cell viability by MTT assay. The values are presented as the means $\pm \mathrm{SD}(\mathrm{n}=3)$. ${ }^{*} \mathrm{P}<0.05$ versus kaempferol alone sample.

p53, phosphorylation of ATM and phosphorylation of p53 protein levels (Fig. 9). We used the specific inhibitors that include Z-IETD-FMK (a specific caspase- 8 inhibitor), $\mathrm{N}$-acetylcysteine (NAC, an antioxidant), caffeine (an ATM kinase inhibitor) and p53 siRNA to confirm this pathway. We found that kaempferol triggered HUVEC apoptosis through the ROS-mediated ATM/p53 signaling.

The p53 tumor suppressor protein is an essential regulator in controlling cell growth and cell death $(6,20)$. In response to intracellular and extracellular stress, p53 is activated and serves as a transcription factor that orchestrates various targets, which in turn modulate multitude of cellular processes such as DNA repair, cell cycle arrest and apoptosis $(28,29)$. It is reported that p53-inducible pro-apoptotic genes trigger apoptosis through both the extrinsic and the intrinsic apoptotic molecular pathways (30). Our results showed that kaempferol significantly increased ROS production (Fig. 7A) and the protein levels of Fas/CD95, DR4, DR5, ATM, p-ATM (Ser1981), p53, and p-p53 (Ser15) in HUVECs (Fig. 8). In addition, knockdown of p53 expression by p53 siRNA significantly inhibited the cell growth inhibitory effects (Fig. 9A) after treatment with kaempferol in HUVECs. Based on our results, we suggest that p53 might be involved in kaempferol-upregulated death receptor signaling.

In addition to the death receptor pathway, our results suggest that kaempferol induced apoptosis through mitochondriadependent pathway. The elevation of $\operatorname{DiOC}_{6}(3)$ fluorescence indicated the loss of $\Delta \Psi \mathrm{m}$ in kaempferol-treated HUVECs (Fig. 4B). The dissipation of $\Delta \Psi \mathrm{m}$ is attributed to the opening of mitochondrial permeability transition (MTP) pore. Hence, 


\section{Kaempferol Death receptor pathway}

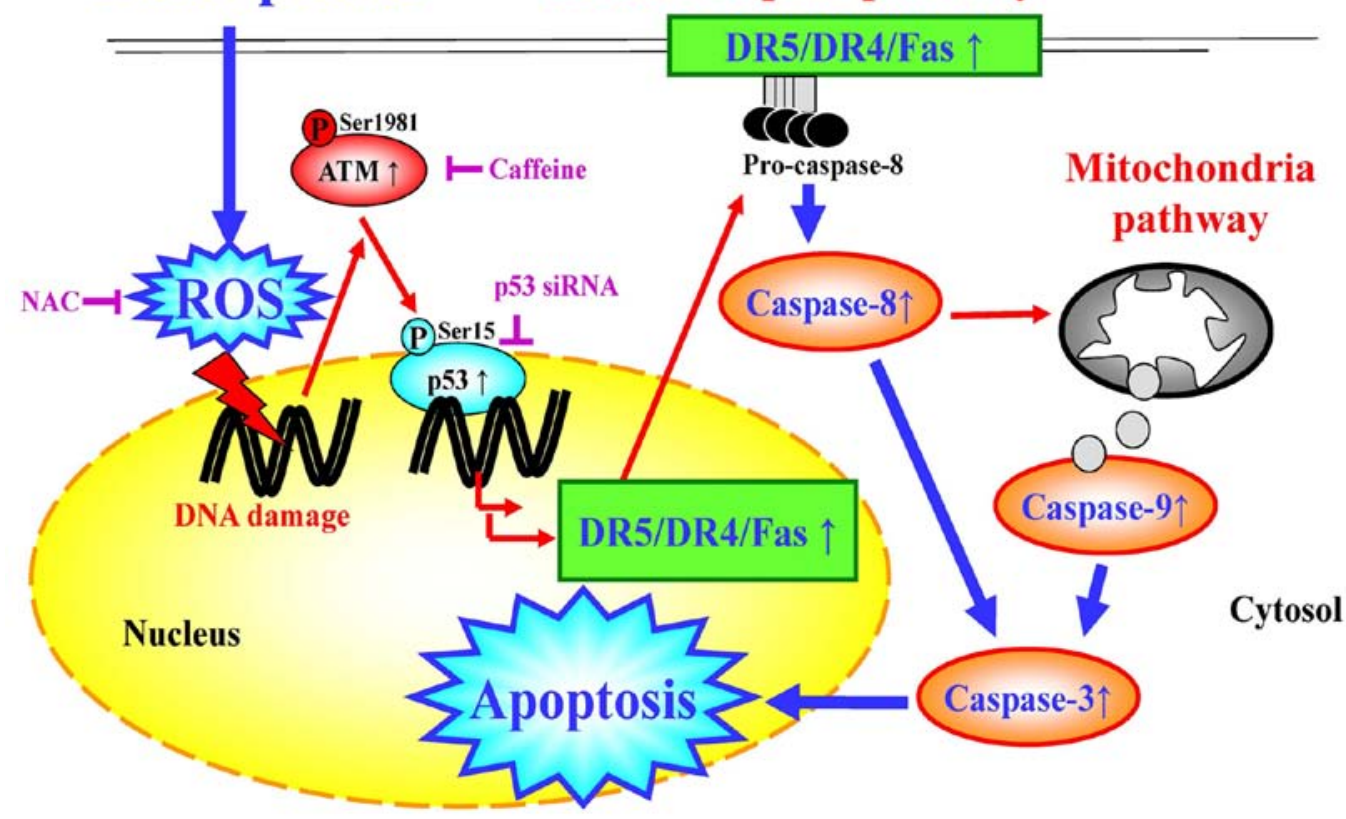

Figure 10. A proposed model for the action and possible signaling pathways of kaempferol on HUVECs. Kaempferol induces apoptosis through both extrinsic and intrinsic apoptotic pathways, resulting from p53-mediated ATM/Fas/DR4/DR5 signaling, which counteracts the induction of apoptotic death in HUVECs.

we suggest that kaempferol led to the persistent opening of the MTP pore, which resulted in mitochondrial swelling and the rupture of mitochondrial outer membrane, ultimately the release of intermembrane proteins such as cytochrome $c$, Apaf-1, pro-caspase-9, AIF and Endo G that trigger cell apoptosis $(6,19)$.

Oxidative stress is closely related to cancer and often associated with cancer prevention and cancer therapy agents $(15,31)$. It was report that reactive oxygen species (ROS) not only function as a regulator of subcellular events but are also able to induce cell apoptosis (8). Yang et al (32) demonstrated that kaempferol reduced the glutamate-induced oxidative stress in mousederived hippocampal neuronal HT22 cells. Ondricek et al (33) showed that kaempferol rescued RGC-5 cells from iodoacetic acid-induced cell death, as well as reduced caspase activation and ROS generation. However, Jeong et al (34) demonstrated that kaempferol caused an increase in generation of reactive oxygen species (ROS), and induced cell death in human glioma cells. Kim et al (35) also showed that kaempferol induced the generation of fluorescent DCF in the MCF-7 cells, and treatment with $\mathrm{N}$-acetylcysteine suppressed kaempferol-induced PARP cleavage. Sharma et al (36) also showed kaempferol in glioblastoma cells induced apoptosis through oxidative stress. In this study, kaempferol was found to be less cytotoxic towards HUVECs after pre-treatment with $\mathrm{N}$-acetylcysteine, suggesting that kaempferol induced oxidative stress in HUVECs (Fig. 7B). Based on the result from $\mathrm{H}_{2}$ DCFDA assay, surprisingly kaempferol was found to stimulate the ROS formation in HUVECs.

In conclusion, the molecular signaling pathway in HUVECs caused by kaempferol is summarized in Fig. 10. Our study discovered that kaempferol reduced HUVEC viability and induced DNA damage and DNA fragmentation through activating the levels of caspase-3, -8 , and -9 signaling, which were upregulated by ROS-mediated p53/ATM molecules following stimulations of p53 downstream protein levels of Fas/CD95, DR4, and DR5. Our results suggest that kaempferol warrants further development as an anti-angiogenetic agent.

\section{Acknowledgements}

This study was financially supported by research grants from Kaohsiung Veterans General Hospital Pingtung Branch, Pingtung, Taiwan.

\section{References}

1. Padma VV: An overview of targeted cancer therapy. Biomedicine (Taipei) 5: 19, 2015.

2. Wang J, Li G, Wang Y, Tang S, Sun X, Feng X, Li Y, Bao G, Li P, Mao X, et al: Suppression of tumor angiogenesis by metformin treatment via a mechanism linked to targeting of HER2/HIF-1 $\alpha /$ VEGF secretion axis. Oncotarget 6: 44579-44592, 2015.

3. He MF, Gao XP, Li SC, He ZH, Chen N, Wang YB and She JX: Anti-angiogenic effect of auranofin on HUVECs in vitro and zebrafish in vivo. Eur J Pharmacol 740: 240-247, 2014.

4. Beedie SL, Peer CJ, Pisle S, Gardner ER, Mahony C, Barnett S, Ambrozak A, Gütschow M, Chau $\mathrm{CH}$, Vargesson $\mathrm{N}$, et al: Anticancer properties of a novel class of tetrafluorinated thalidomide analogues. Mol Cancer Ther 14: 2228-2237, 2015.

5. Liang CH, Wang GH, Chou TH, Wang SH, Lin RJ, Chan LP, So EC and Sheu JH: 5-epi-Sinuleptolide induces cell cycle arrest and apoptosis through tumor necrosis factor/mitochondriamediated caspase signaling pathway in human skin cancer cells. Biochim Biophys Acta 1820: 1149-1157, 2012.

6. Chiang JH, Yang JS, Lu CC, Hour MJ, Chang SJ, Lee TH and Chung JG: Newly synthesized quinazolinone HMJ-38 suppresses angiogenetic responses and triggers human umbilical vein endothelial cell apoptosis through p53-modulated Fas/death receptor signaling. Toxicol Appl Pharmacol 269: 150-162, 2013.

7. Ray PD, Huang BW and Tsuji Y: Reactive oxygen species (ROS) homeostasis and redox regulation in cellular signaling. Cell Signal 24: 981-990, 2012.

8. Indran IR, Tufo G, Pervaiz S and Brenner C: Recent advances in apoptosis, mitochondria and drug resistance in cancer cells. Biochim Biophys Acta 1807: 735-745, 2011. 
9. Tsai AC, Pan SL, Sun HL, Wang CY, Peng CY, Wang SW, Chang YL, Kuo SC, Lee KH and Teng CM: CHM-1, a new vascular targeting agent, induces apoptosis of human umbilical vein endothelial cells via p53-mediated death receptor 5 up-regulation. J Biol Chem 285: 5497-5506, 2010

10. Chiu YJ, Hour MJ, Lu CC, Chung JG, Kuo SC, Huang WW, Chen HJ, Jin YA and Yang JS: Novel quinazoline HMJ-30 induces U-2 OS human osteogenic sarcoma cell apoptosis through induction of oxidative stress and up-regulation of ATM/ p53 signaling pathway. J Orthop Res 29: 1448-1456, 2011.

11. Huang WW, Tsai SC, Peng SF, Lin MW, Chiang JH, Chiu YJ, Fushiya S, Tseng MT and Yang JS: Kaempferol induces autophagy through AMPK and AKT signaling molecules and causes G2/M arrest via downregulation of CDK1/cyclin B in SK-HEP-1 human hepatic cancer cells. Int J Oncol 42: 2069-2077, 2013.

12. Chen AY and Chen YC: A review of the dietary flavonoid, kaempferol on human health and cancer chemoprevention. Food Chem 138: 2099-2107, 2013.

13. Huang WW, Chiu YJ, Fan MJ, Lu HF, Yeh HF, Li KH, Chen PY, Chung JG and Yang JS: Kaempferol induced apoptosis via endoplasmic reticulum stress and mitochondria-dependent pathway in human osteosarcoma U-2 OS cells. Mol Nutr Food Res 54: $1585-1595,2010$

14. Chen HJ, Lin CM, Lee CY, Shih NC, Peng SF, Tsuzuki M, Amagaya S, Huang WW and Yang JS: Kaempferol suppresses cell metastasis via inhibition of the ERK-p38-JNK and AP-1 signaling pathways in U-2 OS human osteosarcoma cells. Oncol Rep 30: 925-932, 2013.

15. Lu CC, Chen HP, Chiang JH, Jin YA, Kuo SC, Wu TS, Hour MJ, Yang JS and Chiu YJ: Quinazoline analog HMJ-30 inhibits angiogenesis: Involvement of endothelial cell apoptosis through ROS-JNK-mediated death receptor 5 signaling. Oncol Rep 32: 597-606, 2014.

16. Lu CC, Yang SH, Hsia SM, Wu CH and Yen GC: Inhibitory effects of Phyllanthus emblica L. on hepatic steatosis and liver fibrosis in vitro. J Funct Foods 20: 20-30, 2016.

17. Lu CC, Yang JS, Chiang JH, Hour MJ, Lin KL, Lee TH and Chung JG: Cell death caused by quinazolinone HMJ-38 challenge in oral carcinoma CAL 27 cells: Dissections of endoplasmic reticulum stress, mitochondrial dysfunction and tumor xenografts. Biochim Biophys Acta 1840: 2310-2320, 2014.

18. Yang JS, Hour MJ, Huang WW, Lin KL, Kuo SC and Chung JG: MJ-29 inhibits tubulin polymerization, induces mitotic arrest, and triggers apoptosis via cyclin-dependent kinase 1-mediated Bcl-2 phosphorylation in human leukemia U937 cells. J Pharmacol Exp Ther 334: 477-488, 2010.

19. Lu CC, Huang BR, Liao PJ and Yen GC: Ursolic acid triggers nonprogrammed death (necrosis) in human glioblastoma multiforme DBTRG-05MG cells through MPT pore opening and ATP decline. Mol Nutr Food Res 58: 2146-2156, 2014.

20. Haupt S, Berger M, Goldberg Z and Haupt Y: Apoptosis - the p53 network. J Cell Sci 116: 4077-4085, 2003.

21. Calderón-Montaño JM, Burgos-Morón E, Pérez-Guerrero C and López-Lázaro M: A review on the dietary flavonoid kaempferol. Mini Rev Med Chem 11: 298-344, 2011
22. Cho HJ and Park JH: Kaempferol induces cell cycle arrest in HT-29 human colon cancer cells. J Cancer Prev 18: 257-263, 2013.

23. Zhang Q, Cheng G, Qiu H, Zhu L, Ren Z, Zhao W, Zhang T and Liu L: The p53-inducible gene 3 involved in flavonoid-induced cytotoxicity through the reactive oxygen species-mediated mitochondrial apoptotic pathway in human hepatoma cells. Food Funct 6: 1518-1525, 2015.

24. Song H, Bao J, Wei Y, Chen Y, Mao X, Li J, Yang Z and Xue Y: Kaempferol inhibits gastric cancer tumor growth: An in vitro and in vivo study. Oncol Rep 33: 868-874, 2015.

25. Dang Q, Song W, Xu D, Ma Y, Li F, Zeng J, Zhu G, Wang X, Chang LS, He D, et al: Kaempferol suppresses bladder cancer tumor growth by inhibiting cell proliferation and inducing apoptosis. Mol Carcinog 54: 831-840, 2015

26. Kim JD, Liu L, Guo W and Meydani M: Chemical structure of flavonols in relation to modulation of angiogenesis and immuneendothelial cell adhesion. J Nutr Biochem 17: 165-176, 2006.

27. Zhao X, Song JL, Kim JD, Lee JS and Park KY: Fermented $\mathrm{Pu}$-erh tea increases in vitro anticancer activities in HT-29 cells and has antiangiogenetic effects on HUVECs. J Environ Pathol Toxicol Oncol 32: 275-288, 2013.

28. Jänicke RU, Sohn D and Schulze-Osthoff K: The dark side of a tumor suppressor: Anti-apoptotic p53. Cell Death Differ 15: 959-976, 2008

29. Tor YS, Yazan LS, Foo JB, Wibowo A, Ismail N, Cheah YK, Abdullah R, Ismail M, Ismail IS and Yeap SK: Induction of apoptosis in MCF-7 cells via oxidative stress generation, mitochondria-dependent and caspase-independent pathway by ethyl acetate extract of Dillenia suffruticosa and its chemical profile. PLoS One 10: e0127441, 2015.

30. Kuribayashi K, Finnberg N, Jeffers JR, Zambetti GP and El-Deiry WS: The relative contribution of pro-apoptotic p53-target genes in the triggering of apoptosis following DNA damage in vitro and in vivo. Cell Cycle 10: 2380-2389, 2011.

31. Reuter S, Gupta SC, Chaturvedi MM and Aggarwal BB: Oxidative stress, inflammation, and cancer: How are they linked? Free Radic Biol Med 49: 1603-1616, 2010.

32. Yang EJ, Kim GS, Jun M and Song KS: Kaempferol attenuates the glutamate-induced oxidative stress in mouse-derived hippocampal neuronal HT22 cells. Food Funct 5: 1395-1402, 2014

33. Ondricek AJ, Kashyap AK, Thamake SI and Vishwanatha JK: A comparative study of phytoestrogen action in mitigating apoptosis induced by oxidative stress. In Vivo 26: 765-775, 2012.

34. Jeong JC, Kim MS, Kim TH and Kim YK: Kaempferol induces cell death through ERK and Akt-dependent down-regulation of XIAP and survivin in human glioma cells. Neurochem Res 34: 991-1001, 2009.

35. Kim BW, Lee ER, Min HM, Jeong HS, Ahn JY, Kim JH, Choi HY, Choi H, Kim EY, Park SP, et al: Sustained ERK activation is involved in the kaempferol-induced apoptosis of breast cancer cells and is more evident under 3-D culture condition. Cancer Biol Ther 7: 1080-1089, 2008.

36. Sharma V, Joseph C, Ghosh S, Agarwal A, Mishra MK and Sen E: Kaempferol induces apoptosis in glioblastoma cells through oxidative stress. Mol Cancer Ther 6: 2544-2553, 2007. 